\title{
Mathematical modeling of first order process with dead time using various tuning methods for industrial applications
}

\author{
Sathish Kumar Shanmugam¹, Yuvaraj Duraisamy², Meenakumari Ramachandran², \\ Senthilkumar Arumugam ${ }^{4}$ \\ ${ }^{1}$ Jansons Institute of Technology, Coimbatore, Tamilnadu, India \\ ${ }^{2}$ Tamilnadu College of Engineering, Coimbatore, Tamilnadu, India \\ ${ }^{3}$ Kongu Engineering College, Erode, Tamilnadu, India \\ ${ }^{4}$ Chettinad College of Engineering and Technology, Karur, Tamilnadu, India \\ ${ }^{1}$ Corresponding author \\ E-mail: ${ }^{1}$ ssk@jit.ac.in, ${ }^{2}$ pciyuvaraj@gmail.com, ${ }^{3}$ oremkay@gmail.com, ${ }^{4}$ bask2k1@yahoo.co.in
}

Received 12 December 2018; received in revised form 2 January 2019; accepted 27 January 2019 DOI https://doi.org/10.21595/mme.2019.20447

Check for updates

Copyright (C) 2019 Sathish Kumar Shanmugam, et al. This is an open access article distributed under the Creative Commons Attribution License, which permits unrestricted use, distribution, and reproduction in any medium, provided the original work is properly cited.

\begin{abstract}
The proposed research involves Mathematical modeling for first order process with dead time using various tuning methods for industrial applications. Different tuning methods are proposed. Proposed method selection depends on plant operating conditions and also depending upon the process dynamics. The PID controller is most widely used for industrial process control. Modeling were developed for modified internal control model [4-6] in this proposed research. The proposed work is the modeling and simulation of three different first order processes with dead time. The standard controller tuning method is used to obtain the steady state response of first order with dead time.
\end{abstract}

Keywords: IMC, tuning, transfer function, simulation, analysis.

\section{Introduction}

The preferred output of a control system is obtained by tuning the controller parameters according to the set point. The first order system is widely used in industrial control system to represent the behavior of process dynamics $[1,2]$. The delay takes place in process measurement, controller action, actuator operation and computation is called delay time. Dead time is the time taken by the system to produce the response after the input is applied [3].

\section{Block diagram of feedback control system}

Fig. 1 shows the block diagram proposed IMC Feedback Control System. The algorithm is necessity oe for tuning. The control action is done by the actuator. The input signal which is attenuated will control the parameter. Sensor is used for controlling the signal measure the process variable from the process output. The control algorithm is used to nullify the error signal to zero [4-6].

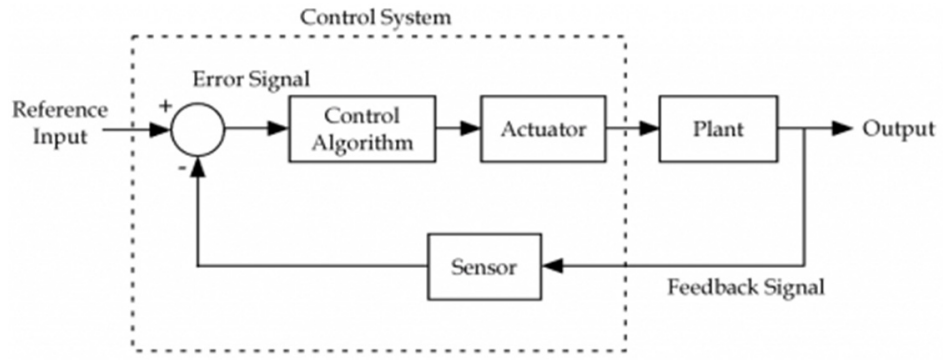

Fig. 1. Block diagram of proposed IMC feedback control system 


\section{Literature review}

- Astrom and Haggland proposed an alternative method of the system. The technique itself create its own test signal based on the estimation [7].

- The robustness of the automatic tuning of controller by Zeigler's technique is improved by Astrom and Haggland method. In this robust loop shaping rule called MIGO and AMIGO is introduced. MIGO concentrates on maximizes the integral gain to improve the robustness of the system.

- Wallen et al., enhanced the loop shaping based tuning by introducing an additional constraint the ratio of integral time to the derivative time for tuning the PID controller. And also, it makes some conditions the curvature should be negative and it should have a decreasing phase of a curve.

- Zeigler and Nichols proposed the first auto tuning procedure for controllers. The sensitivity, Reset rate and pre-act time are the characteristics of the reaction curve for designing an automatic controller $[8,9]$.

\section{Materials and methods}

\subsection{Controller tuning}

When PID controller has been selected, Different methods are required for tuning. This is called as 'controller tuning'. Different methods adopted for the proposed tuning are analysed and its results were carried out with the modified structure. The method proposed is Internal Model Control (IMC).

$\mathrm{r}(\mathrm{s})$

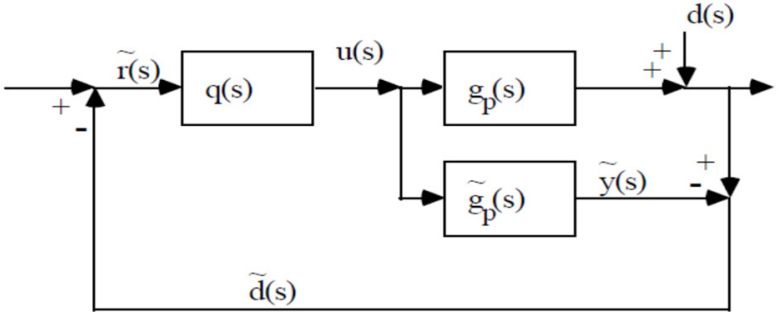

Fig. 2. Proposed IMC schematic circuit

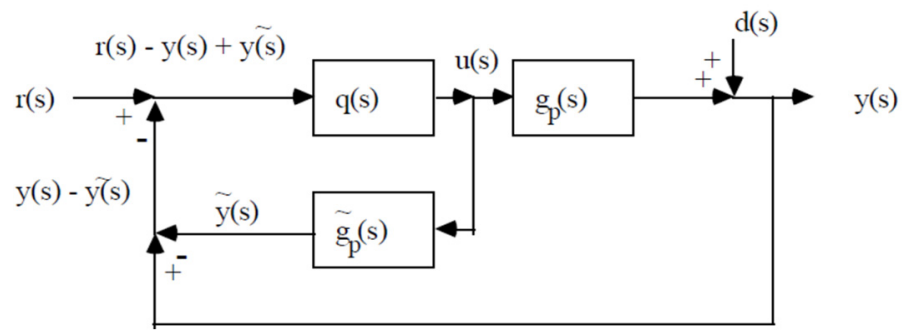

Fig. 3. Proposed IMC schematic circuit with surface change

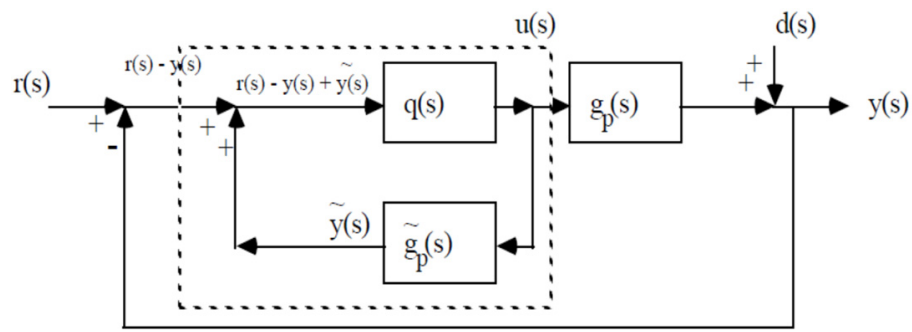

Fig. 4. Modified proposed IMC schematic circuit with surface change 


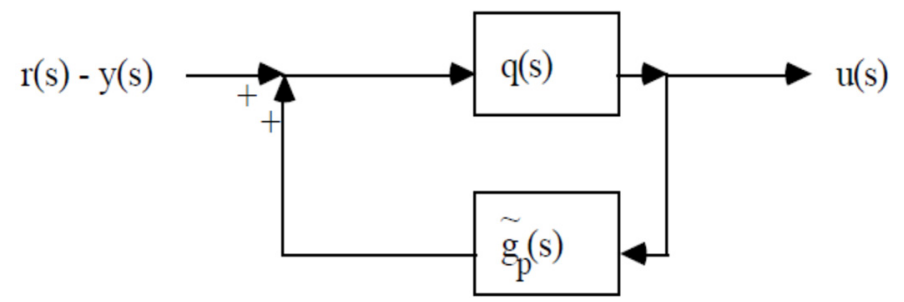

Fig. 5. Modified proposed IMC schematic circuit with surface change inner loop

The standard feedback of proposed IMC circuit is given by:

$g_{c}(s)=\frac{q(S)}{1-g_{p}(s) q(S)}$

The equivalent circuit equation of proposed structure IMC is given by:

$g_{c}(s)=\frac{q(S)}{1-\tilde{g}_{p}(s) q(S)}$.

The PID controller tuning equation is given by:

$g_{c}(s)=k_{c}\left[\frac{\tau_{1} \tau_{D} s^{2}+\tau_{1} s+1}{\tau_{1} s}\right]\left[\frac{1}{\tau_{F} s+1}\right]$.

The proposed method first order process is given by:

$\tilde{g}_{p}(s)=\frac{k_{p}}{\tau_{p} s+1}$.

After filtering in the process is given by:

$q(s)=\tilde{q}(s) f(s)=g_{p}{ }^{-1}(s) f(s)=\left[\frac{\tau_{p} s+1}{k_{p}}\right]\left[\frac{1}{\lambda_{s}+1}\right], q(s)=\frac{1}{k_{p}} \frac{\tau_{p} s+1}{\lambda_{s}+1}$,

After transforming the equation can be written as:

$g_{c}(s)=\frac{q(S)}{1-\tilde{g}_{p}(s) q(S)}=\frac{\frac{\tau_{p} s+1}{k_{p}\left(\lambda_{s}+1\right)}}{1-\frac{k_{p}}{\tau_{p} s+1} \frac{\tau_{p} s+1}{k_{p}\left(\lambda_{s}+1\right)}}=\frac{\tau_{p} s+1}{k_{p} \lambda_{s}}$.

Multiplying with tuning parameters:

$g_{c}(s)=\left[\frac{\tau_{p}}{k_{p} \lambda}\right] \frac{\tau_{p} s+1}{\tau_{p} s}$.

Similarly, for second order process:

$g_{c}(s)=\left[\frac{\tau_{1}+\tau_{2}}{k_{p} \lambda}\right]\left[\frac{\tau_{1} \tau_{2} s^{2}+\left(\tau_{1}+\tau_{2}\right) s+1}{\left(\tau_{1}+\tau_{2}\right) s}\right]$. 


\subsection{Methods of tuning for servo response}

The controller tuning is performed to stabilize the process output according to the set point changes. The feedback controller is used for setting point tracking. The error signal is generated which is the differences between setpoint and process variable. The controller tuning is based on the error signal and it is performed to nullify the error. The controller tuning is performed to stabilize the process output according to the load disturbance. The feedback controller is not suitable for load disturbance tracking. The advanced control mechanism such as feedforward controller is used to track the load disturbance. The feedforward controller takes control action according to the load changes.

\subsubsection{Cohen-Coon tuning}

This method is also based on a delayed first order rise, and the method tunes the PID gains to achieve quarter-amplitude damping, i.e. each peak in the transient is one quarter of the immediate preceding peak. If the unit step response of the open loop plant is available, then process gain $(K)$, dead time $\left(t_{d}\right)$ and time constant $(T)$ can be determined. PID controllers are designed to test the system and selected based on the performance and selection criteria. Table 1 shows the Cohen Coon method.

Table 1. Cohen Coon

\begin{tabular}{|c|c|c|c|}
\hline Controller type & $\left(K_{C}\right)$ & $T_{I}$ & $T_{D}$ \\
\hline PID & $\frac{1.49}{k_{m} \tau_{m}}$ & $2 \tau_{m}$ & $1.27 \tau_{m}$ \\
\hline
\end{tabular}

\subsubsection{Ziegler Nichols tuning}

Ziegler Nichols method is also called as Ultimate Cycle Method. It is based on adjusting a closed loop until steady state oscillations occur. This method is used to minimizing the total error of the response. To return to desired level operation as soon as possible and to keep the maximum deviation as small as possible this method is used. The integral absolute error is the integral error which defines the stability of the system. Larger the integral error, smaller the stability is. Thus, by minimizing the integral absolute error we get the good robustness and performance. Thus, IAE is also taken measuring parameter. Control objectives focus on the behavior of the system such as to produce a zero steady state error, a fast transient response to a step command, a short settling time and low overshoot. It is also desirable to make the system less sensitive to disturbance. The period of these oscillations is defined as the ultimate period $P_{u}$. The controller gains at which these oscillations occur is referred to as the ultimate gain $K_{u}$. Ziegler Nichols method are shown in Table 2.

Table 2. Ziegler Nichols method

\begin{tabular}{|c|c|c|c|}
\hline Controller type & $K_{C}$ & $T_{I}$ & $T_{D}$ \\
\hline PID Zeigler Nichol's & $\frac{K_{u}}{1.7}$ & $\frac{P_{u}}{2}$ & $\frac{P_{u}}{8}$ \\
\hline $\begin{array}{c}\text { Ultimate cycle PID } \\
\text { developed by mc Millan }\end{array}$ & $1.009 \frac{T m}{k m \tau_{m}}\left\{\frac{1}{1+\left(\frac{T m}{\tau_{m}}\right)^{.65}}\right\}^{2}$ & $2 \tau_{m}\left\{1+\left(\frac{T m}{\tau_{m}}\right)^{.65}\right\}$ & $0.26 T_{I}$ \\
\hline
\end{tabular}

\subsubsection{FOPTD transfer functions}

The PID tuning formulas are applied for the three FOPTD transfer functions to obtain the analysis. The FOPTD transfer function is considered based on ratio of dead-time to the time 
constant less than one, equal to 1 and greater than 1 . Z-N method is time consuming and forces the system to margin if instability. Many other algorithms have been proposed to solve these problems by obtaining critical data (ultimate gain and frequency) under more acceptable conditions. One of these methods is damped oscillation method. Z-N method are shown in Table 3.

Table 3. Z-N method

\begin{tabular}{|c|c|c|c|}
\hline Controller type & $K_{C}$ & $T_{I}$ & $T_{D}$ \\
\hline PID & $K_{u} / 1.3$ & $2.2 P_{u}$ & $P_{u} / 5.2$ \\
\hline
\end{tabular}

The implicit manner based PID control algorithm is simple and robust to handle the model inaccuracies and hence using IMC-PID tuning method a clear trade-off between closed loop performance with damped oscillation and robustness to model inaccuracy is achieved with a single tuning parameter are show in Table 4 for implicit manner.

Table 4. For implicit manner

\begin{tabular}{|c|c|c|c|}
\hline Controller type & $K_{C}$ & $T_{I}$ & $T_{D}$ \\
\hline PID & $2 / K(\lambda+\theta / 2)$ & $2 \lambda+\theta$ & $\frac{\lambda \theta+\left(\theta^{2}\right) / 4}{2 T+\theta}$ \\
\hline
\end{tabular}

\subsubsection{Integrating process transfer functions}

The PID tuning formulas are applied for the three different order transfer functions to obtain the analysis in order to study the integrating process performance. The different order integrating process transfer functions considered for the analysis are.

The PID tuning formulas are applied for the three different order transfer functions to obtain the analysis in order to study the integrating process performance. The different order integrating process transfer functions considered for the analysis are,

(i) Transfer function 1.

The pure integrator process transfer function with low delay consider as example of benchmark process default value of second order system for controlling:

$G_{p 1}(s)=\frac{1}{s} e^{-2 s}$

(ii) Transfer function 2.

The pure integrator process transfer function with low delay consider as example of benchmark process default value of second order system for controlling:

$G_{p 2}(s)=\frac{1}{s} e^{-5 s}$

(iii) Transfer function 3.

The higher order integrator process transfer function without delay consider as example of benchmark process default value of second order system for controlling:

$G_{p 3}(s)=\frac{(0.17 s+1)^{2}}{s(s+1)^{2}(0.028 s+1)}$.

The transfer function is approximated to integrator process with delay by using half rule approximation in order to work with, and the transfer function is example of benchmark process default value of second order system for controlling: 
$G_{p 3}(s)=\frac{1}{s} e^{-1.69 s}$

\section{Results and discussions}

\subsection{MATLAB software for simulation of FOPDT}

Simulink is widely used for analysis of first order process with dead time. The Simulink is used in this proposed work to obtain the response of first order with dead time. Simulink block diagram of FOPTD set point and transfer function for load disturbance are shown in Figs. 6 and 7.

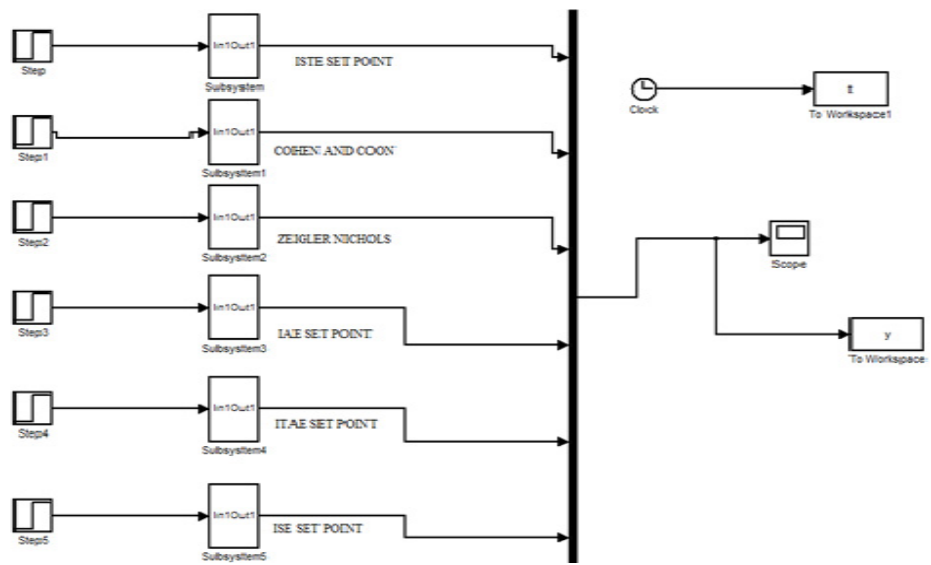

Fig. 6. Simulink block diagram for FOPTD for set point

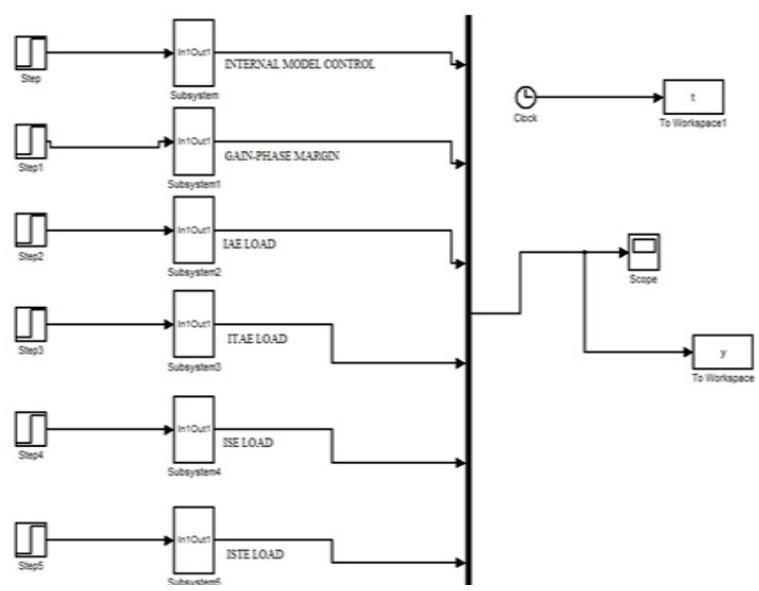

Fig. 7. Simulink block diagram of FOPTD transfer function for load disturbance

\subsubsection{Transfer function 1}

The ratio of time delay $\theta$ to the time constant $\tau$ is less than one:

$G_{p 1}(s)=\frac{1}{5 s+1} e^{-s}$

Fig. 8 shows the output of transfer function 1. Fig. 11 shows the output of transfer function 1 setpoint. 


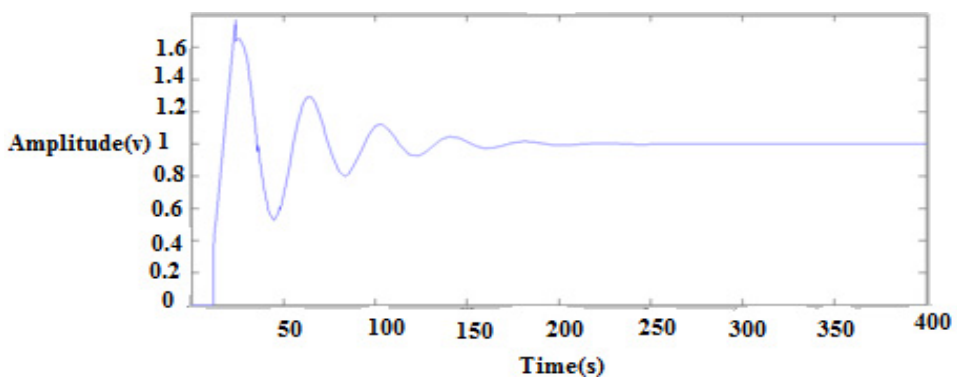

Fig. 8. Output of transfer function 1

\subsubsection{Transfer function 2}

The ratio of time delay $\theta$ to the time constant $\tau$ is unity:

$G_{p 2}(s)=\frac{4}{10 s+1} e^{-10 s}$

Fig. 9 shows the output of transfer function 2. Fig. 12 shows the output of transfer function 2 setpoint.

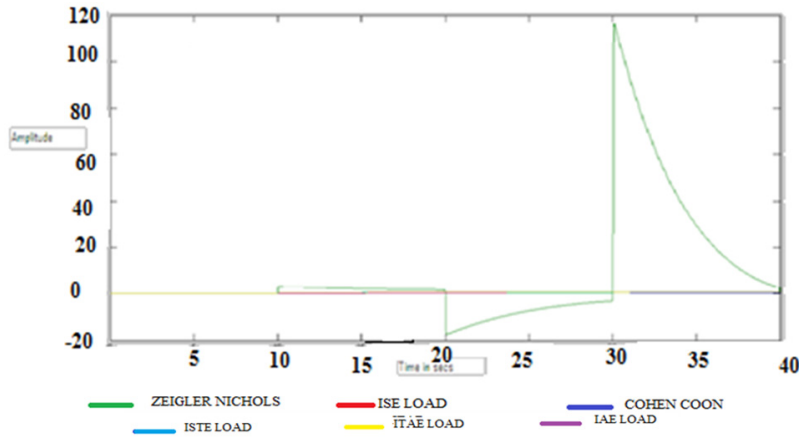

Fig. 9. Output of transfer function 2

\subsubsection{Transfer function 3}

The ratio of time delay $\theta$ to the time constant $\tau$ is greater than one:

$G_{p 3}(s)=\frac{1}{7 s+1} e^{-14 s}$

Fig. 10 shows the output of transfer function 3. Fig. 13 shows the output of transfer function 3 setpoint.

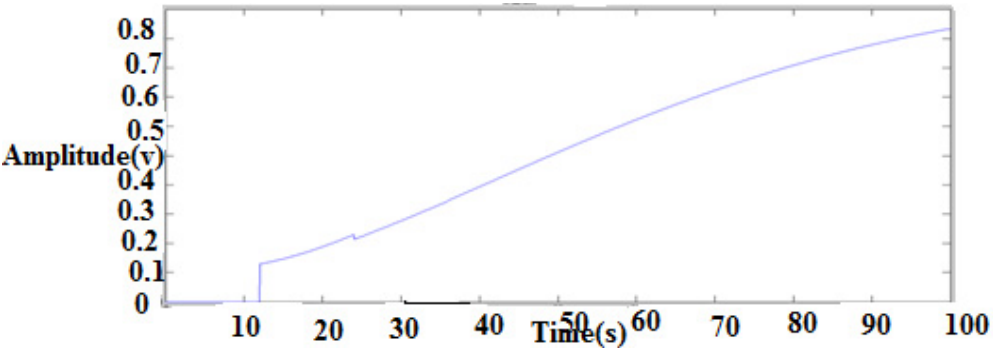

Fig. 10. Output of transfer function 3 


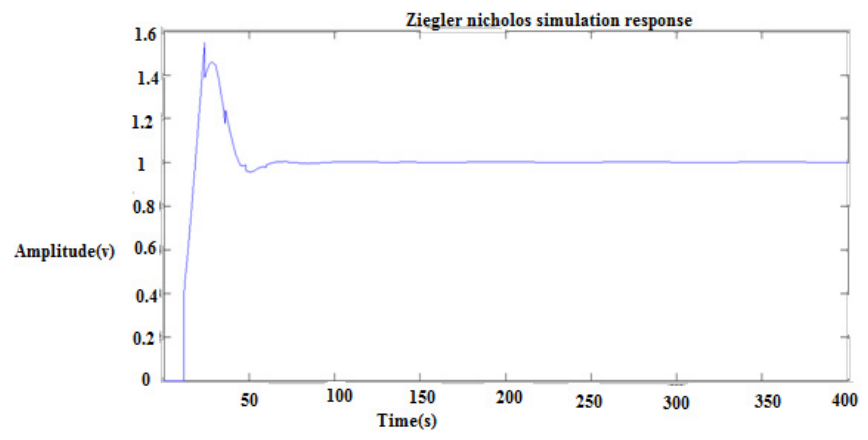

Fig. 11. Output of transfer function 1 (set point)

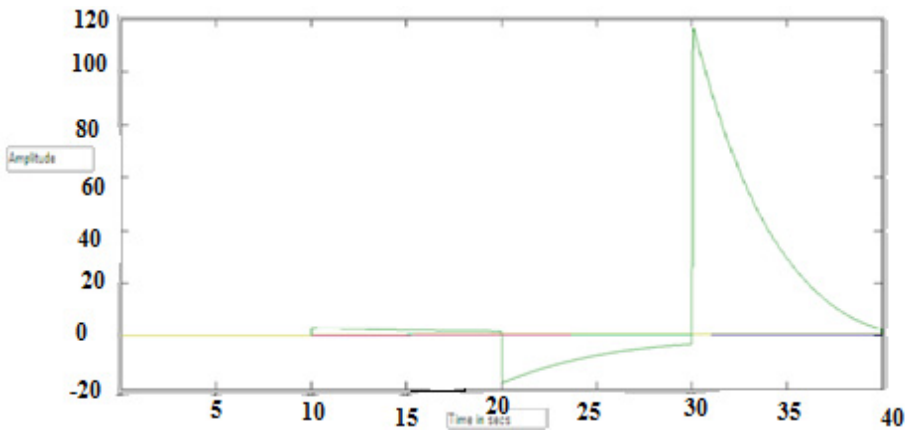

Fig. 12. Output of transfer function 2 (set point)

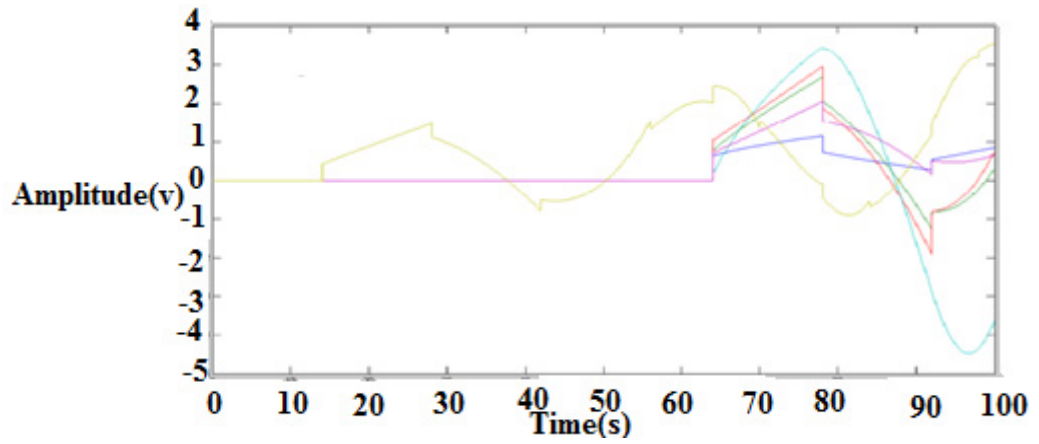

Fig. 13. Output of transfer function 3 (set point)

\subsection{Simulation results of closed loop methods}

The various output loop response is shown in Figs. 14 to 18.

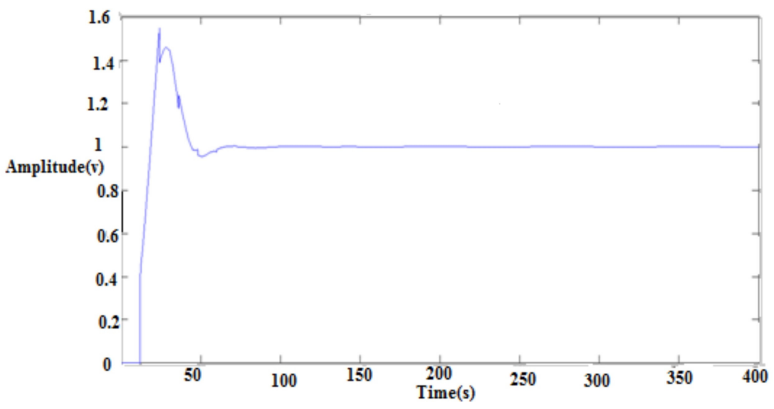

Fig. 14. Ziegler Nichols closed loop response 


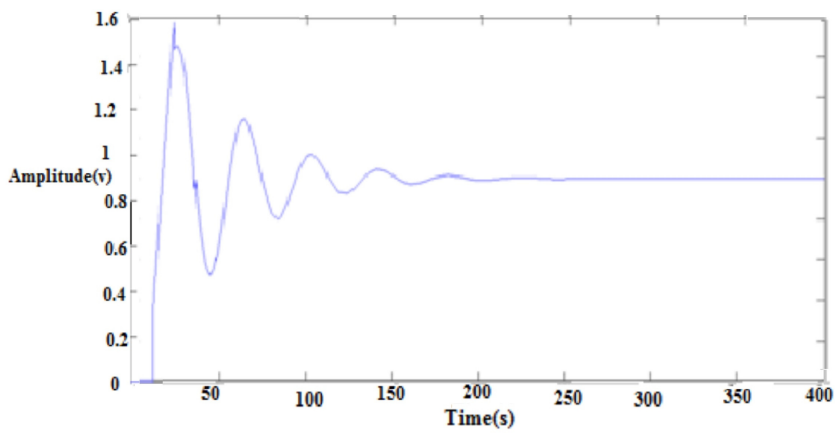

Fig. 15. IMC closed loop response

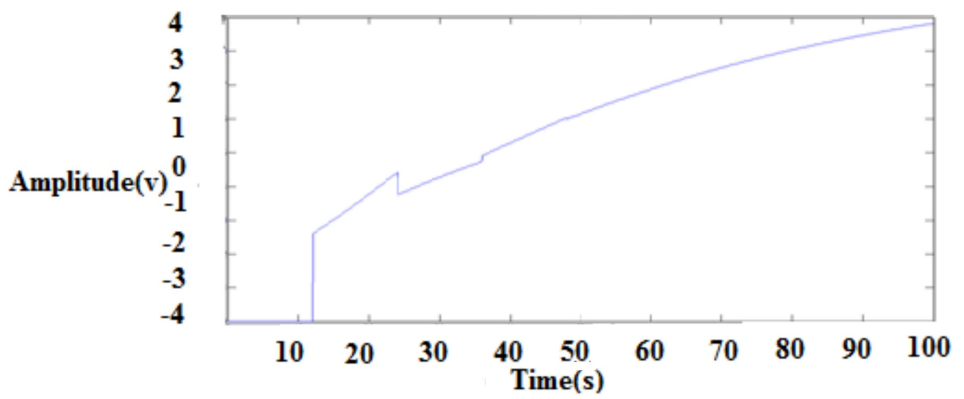

Fig. 16. Integral of square error closed loop response

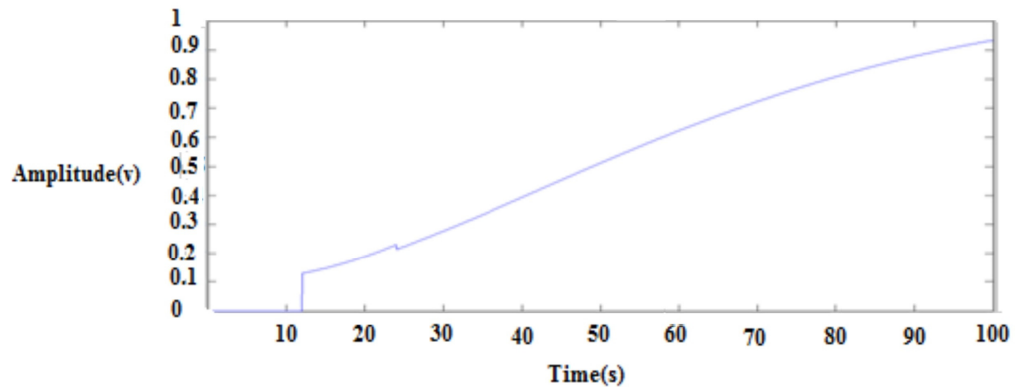

Fig. 17. Integral of absolute error closed loop response

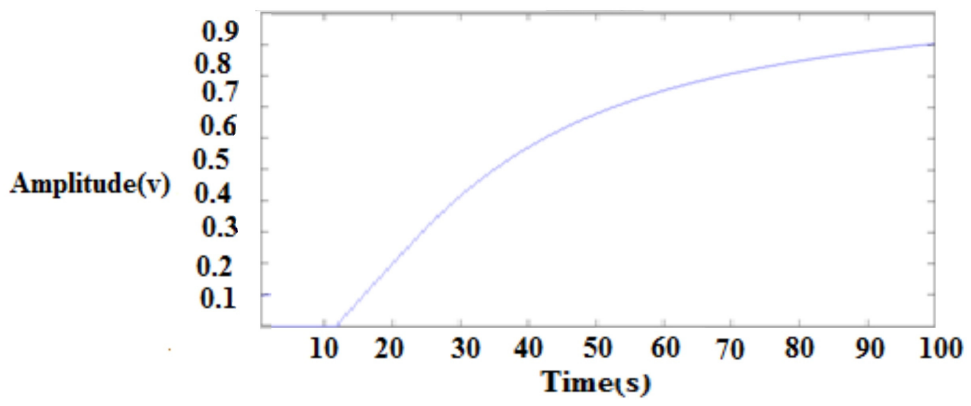

Fig. 18. Integral of square time error closed loop response

\section{Conclusions}

The first order with delay process is widely used to represent the process dynamics. The selection of controller and tuning methods are important consideration in the control system design. This proposed work is the tuning and analysis of three different first order with dead time. 
This analysis shows that the performance is best for the ratio less than 1 for Zeigler Nichols, Cohen coon, ITAE load disturbances and IMC, ITAE, ISE, ISTE, GP set point method. This model produces a better transient response. This proposed work can be extended by using Fuzzy Logic Controller for first order with dead time and performance characteristics can be compared.

\section{References}

[1] Ozipneci Burak, Tolbert Leon M. Simulink implementation of induction machine model - a modular approach. IEEE International Electric Machines and Drives Conference, 2003.

[2] Dorf C. R., Bishop R. F. Modern Control System. 10th Edition, Prentice Hall, 2005.

[3] Dumitrescu A., Fodor D., Jokinen T., Rosu M., Bucurencio S. Modeling and simulation of electric drive system using Matlab/Simulink environment. International Conference on Electric Machines and Drives (IEMD), 1999, p. 451-453.

[4] Le Huy H. Modeling and simulation of electrical drives using Matlab/Simulink and power system block set. 27th Annual Conference of the IEEE Industrial Electronics Society, 2001.

[5] Nizam Kamarudin M., Rozali Sahazati Md. Implementation of cascade control DC motor. International Conference on Power and Energy, 2008.

[6] Sondhi S., Hote Y. V. Fractional order PID controller for load frequency control. Energy Conversion and Management, Vol. 85, 2014, p. 343-353.

[7] Debbarma S., Dutta A. Utilizing electric vehicles for LFC in restructured power systems using fractional order controller. IEEE Transactions on Smart Grid, Vol. 8, Issue 6, 2017, p. 2554-2564.

[8] De Keyser R., Muresan C. I., Ionescu C. M. A novel auto-tuning method for fractional order PI/PD controllers. ISA Transactions, Vol. 62, 2016, p. 268-275.

[9] Moosavi S. H. S., Bardsiri V. K. Satin bowerbird optimizer: a new optimization algorithm to optimize ANFIS for software development effort estimation. Engineering Applications of Artificial Intelligence, Vol. 60, 2017, p. 1-15. 